\title{
The Ontogeny of Somatic Stem Cells
}

\author{
Vincent Tropepe $\cdot$ Kursad Turksen
}

Published online: 15 April 2012

(C) Springer Science+Business Media, LLC 2012

\section{Introduction}

Cells in vertebrate tissues are richly diversified in their morphological, biochemical and functional characteristics. Understanding the generation of such an array of cell diversity from relatively few precursor cells continues to be a major research focus in the developmental and stem cell biology fields.

The formation of a relatively complex vertebrate embryo from a simple ball or sheet of primitive cells in early development begins with the process of gastrulation [1]. What emerges during this epoch of development is an embryonic body plan with three separate germ layers: endoderm, mesoderm and ectoderm. These germ layers often appear cytologically indistinct yet among them are the primordial cells that will go on to generate the many distinct tissues of the body.

We generally refer to these primordial cells as 'tissuespecific' or 'somatic' stem cells and we ascribe three main properties to them: self-renewal, tissue-specific multipotentiality, and longevity [2-4]. Major efforts towards defining the unique properties of somatic stem cells has yielded significant insight into the identity and behavior of these cells, in the form of stem cell specific markers and their lineage potential. Nonetheless, there are some fundamental

\footnotetext{
V. Tropepe $(\triangle)$

Department of Cell \& Systems Biology, University of Toronto, 25 Harbord Street,

Toronto, ON M5S 3G5, Canada

e-mail: v.tropepe@utoronto.ca

K. Turksen

Ottawa Hospital Research Institute,

Ottawa, Canada

questions that remain unanswered and we believe this knowledge gap lies at the heart of the challenge to translate what we know about stem cells into successful therapies or biotechnological applications.

In this special issue of Stem Cell Reviews \& Reports, we have asked some of the leading scientists in the developmental biology and stem cell biology fields to comment on a few of these fundamental questions, such as: where do stem cells come from; or, does stem cell behavior change over time, and if so how? These questions exemplify the notion of somatic stem cell ontogeny, which focuses on quantitative and/or qualitative differences in stem cell behavior through time and space. This concept is important for the simple reason that we need to know how fetal stem cells are initially generated in a given tissue, where they are located and whether they maintain the same properties and locations throughout life (e.g., reviews by L. Grabel, and Z. Burke and D. Tosh) $[5,6]$.

Somatic stem cell ontogeny is a concept that deals with some of stem cell biology's more contemporary, and controversial, issues. For instance, the generative processes of somatic stem cells may serve quite distinct purposes at different life stages. Most scientists would agree that during embryogenesis, somatic stem cells have a major and widespread role in tissue formation, yet in adulthood these somatic stem cells are relatively quiescent and typically localized to rather tiny compartments within the same tissues they helped build. In some cases, it has been debated whether bona fide stem cells even exist in adult tissue compartments where they were once active during formative stages [7], while in other cases the debate centers on whether tissues developed to contain only a single stem cell or multiple distinct stem cells that persist in adulthood [8]. It is clear that individual tissues are impacted by predictable physiological variations in growth, maturation, reproduction, and 
senescence, but we don't know if the identity and function of tissue-specific stem cells remains constant during these transitions. Stem cell behavior is also adaptive in response to changes to the animal's physical and chemical environment (i.e. mating and other social interactions, stress, tissue damage, or disease). However, the extent to which these adaptive responses, including regeneration, are the same at different life stages remains poorly understood.

Another issue that is receiving a great deal of attention (and rightly so!) involves the environment in which somatic stem cells reside [9, 10]. Stem cells do not exist in isolation; there is a niche that is required for proper stem cell function. This is because cellular neighbors can help to interpret physiological changes in tissues to enable an appropriate response by stem cells (e.g., review by A. Aziz, S. Sebastian, and J. Dilworth) [11]. Neighboring cells (including stem cell progeny) can also limit the extent to which stem cells proliferate, for example, through feedback inhibition. An emerging view is that the stem cell niche is a major regulator of stem cell behavior in any tissue. Nonetheless, there are many aspects of the niche that remain somewhat mysterious. For instance, do stem cells actively participate in generating and/or maintaining their cellular niche? Are the stem cells that reside with a given niche (either within or between tissues) intrinsically different, or does the niche itself impose the regional specificity of stem cell behavior? Where the presence of more than one tissuespecific stem cell seems plausible, one might ask if this heterogeneity is distributed across niches or co-existing in the same niche. Finally, for all of the above questions, one may also ask how these properties change with age.

One of the more challenging problems with understanding somatic stem cell ontogeny has been to elucidate if common or distinct molecular pathways are used to regulate stem cell behavior (e.g. proliferation) in the embryo compared to the adult [12]. There are now many examples in the literature of particular genes and their associated signaling pathways that are commonly used among various different somatic stem cell populations (e.g. Wnt and Notch signaling immediately come to mind). It is not uncommon, however, to discover that these signaling pathways regulate stem cell behavior differently in fetal tissues compared to their adult counterparts. Part of the challenge also lies in the way we study stem cells. For example, somatic stem cells may express cell surface antigens and respond to growth factors in a fairly uniform manner throughout most life stages. Thus, standard techniques for purifying stem cells and assaying for their behavior in vitro (prospectively or retrospectively) regardless of age have emerged and this might erroneously be interpreted to mean that the stem cells are essentially the same. Few studies have probed further to provide an in depth comparison of gene expression profiles or molecular signaling requirements of tissue-specific stem cells derived from tissues at different ages. These are not trivial experiments, but they are necessary if we are to glean new insight into how stem cell behavior changes throughout life.

Before we can fully benefit from a more refined concept of stem cell ontogeny, we must adopt the fact that stem cells are dynamic. More precisely, stem cell populations are dynamic (e.g., review by T. Tumbar) [13]. We can start with the notion of stem cell mobility. There are certain somatic stem cell populations that might reasonably be considered stationary (e.g. gut epithelial stem cells) and are dependent on highly localized signaling from the niche (e.g., review S. Takashima and V. Hartenstein) [14], whereas others may have limited migratory behavior, such as cortical neural stem cells migrating ventrally to the subventricular zone along the lateral wall of the striatum [15]. Still, other examples of more dramatic migratory capacity come from studies of the hematopoietic stem cells that move throughout the circulatory system and lodge themselves into different tissues (e.g., review by W. Lensch) [16]. We do not know the extent of this behavior among most stem cell populations. For example, is stem cell migration (where present) an adaptation to adult physiology or does it also play a role during development? Stem cell populations are also dynamic in their proliferative behavior. A standard definition for stem cells is the ability for asymmetric self-renewing divisions, yet it has been very difficult to unequivocally demonstrate this phenomenon at the single cell level. Alternatively, new evidence suggests that populations of stem cells can fulfill the requirement for multilineage differentiation and self-renewal by simply balancing the extent of symmetric self-renewal of some stem cells in the population with terminal differentiation by other stem cells in the population [13]. How this process might be regulated at different life stages is not yet clear. As mentioned above, fetal stem cells help to build tissues and so it would seem that expansionary stem cell divisions might predominate during development. In contrast, in most adult tissues where the demand for stem cell contributions is less, the stem cells divide infrequently with the same balanced population dynamic, except perhaps during initial stages of regeneration.

A final thought about stem cell dynamics centers on the nature of stem cell identity. We typically envision a somatic stem cell niche to contain one (or more) stem cell with various stem cell progeny at different stages of differentiation. This lineage relationship is thought to be stable where unique stem cells persist in the niche with age. Is there an alternative to this scenario? What if the stem cell identity can be achieved transiently when required by some cells, as opposed to maintaining a unique and stable stem cell population? The notion of trans-differentiation or dedifferentiation is known to the developmental biology community (especially from the classical embryology literature), but is now permeating through the stem cell biology literature [4]. There are now several examples that would appear 
to challenge our entrenched view of the stem cell lineage hierarchy whereby differentiated (and post-mitotic) cells acquire the capacity to re-enter the cell cycle to generate new cells [17], demonstrating at least some of the properties that are normally ascribed to stem cells, or where stem cell lineages become biased over time [16]. Are these simply rare phenomena or are tissues built and maintained by cells with dynamic cell fate properties? Certainly a more conservative view of somatic stem cell ontogeny would have at its core a stable, identifiable stem cell. Yet, this conceptual framework could be modified to account for cell fate dynamics with a population of cells, perhaps only those associated with a particular niche. Regardless, this is a fascinating area of investigation that will no doubt continue to be explored.

These are a few of the topics that are addressed in this special issue. We commend the contributing authors for providing us with an exceptional collection of review articles. These articles are not only thoroughly informative, but they will engage your intellectual curiosity about some of the outstanding issues related (but certainly not confined) to somatic stem cell ontogeny.

Conflict of Interest Authors declare no conflict of interest

\section{References}

1. Wolpert, L., Beddington, R., Jessell, T., Lawrence, P., Meyerowitz, E., \& Smith, J. (2002) Principles of Development. Oxford University Press.

2. Potten, C. S., \& Loeffler, M. (1990). Stem cells: attributes, cycles, spirals, pitfalls and uncertainties. Lessons from the crypt. Development, 110, 1001-1020.

3. Morrison, S. J., Shah, N. M., \& Anderson, D. J. (1997). Regulatory mechanisms in stem cell biology. Cell, 88, 287-298.
4. Weissman, I. L., Anderson, D. J., \& Gage, F. H. (2001). Stem and progenitor cells: Origins, phenotypes, lineage commitments, and transdifferentiations. Annual Review of Cell and Developmental Biology, 17, 387-403.

5. Grabel, L. (2012). Developmental origin of neural stem cells: The glial cell that could. Stem Cell Reviews. doi:10.1007/s12015-0129349-8.

6. Burke, Z. D., Tosh, D. (2012). Ontogenesis of hepatic and pancreatic stem cells. Stem Cell Reviews. doi:10.1007/s12015-012-9350-

7. Seaberg, R. M., \& van der Kooy, D. (2003). Stem and progenitor cells: the premature desertion of rigorous definitions. Trends in Neurosciences, 26, 125-131.

8. Takeda, N., Jain, R., LeBoeuf, M. R., Wang, Q., Lu, M. M., \& Epstein, J. A. (2011). Interconversion between intestinal stem cell populations in distinct niches. Science, 334, 1420-1424.

9. Lander, A. D., Kimble, J., Clevers, H., Fuchs, E., Montarras, D., Buckingham, M., Calof, A. L., Trumpp, A., \& Oskarsson, T. (2012). What does the concept of the stem cell niche really mean today? BMC Biology, 10, 19.

10. Moore, K. A., \& Lemischka, I. R. (2006). Stem cells and their niches. Science, 311, 1880-1885.

11. Aziz, A., Sebastian S., Dilworth, F.J. (2012) The origin and fate of muscle satellite cells. Stem Cell Reviews. doi:10.1007/s12015-0129352-0.

12. Pietras, E. M., Warr, M. R., \& Passegué, E. (2011). Cell cycle regulation in hematopoietic stem cells. The Journal of Cell Biology, 195, 709-720.

13. Tumbar, T. (2012) Ontogeny and homeostasis of adult epithelial skin stem cells. Stem Cell Reviews. doi:10.1007/s12015-012-9348-9.

14. Takashima, S., Hartenstein, V. (2012) Genetic control of intestinal stem cell specification and development: a comparative view. Stem Cell Reviews. doi:10.1007/s12015-012-9351-1.

15. Willaime-Morawek, S., Seaberg, R. M., Batista, C., Labbe, E., Attisano, L., Gorski, J. A., Jones, K. R., Kam, A., Morshead, C. M., \& van der Kooy, D. (2006). Embryonic cortical neural stem cells migrate ventrally and persist as postnatal stiatal stem cells. The Journal of Cell Biology, 175, 159-168.

16. Lensch, M.W. (2012) An evolving model of hematopoitic stem cell functional identity. Stem Cell Reviews. doi:10.1007/s12015-0129347-X.

17. Dor, Y., Brown, J., Martinez, O. I., \& Melton, D. A. (2004). Adult pancreatic beta-cells are formed by self-duplication rather than stem cell differentiation. Nature, 429, 41-46. 